Rev Biomed 2005; 16:159-167.

\title{
Anatomía del desastre del World Trade Center, Nueva York, E.U.A., desde una perspectiva epidemiológica. ¿Qué debemos aprender de la destrucción urbana masiva?
}

\section{Artículo Original}

Luis M. García-Núñez¹, Ruy Cabello-Pasini², Ricardo Padilla-Solís³ ${ }^{3}$ Pedro Garduño-Manjarrez ${ }^{4}$.

${ }^{1}$ International Visiting Scholar/Research Fellow, Trauma Surgery and Surgical Critical Care, Department of Surgery - Division of Trauma, University of Medicine and Dentistry of New Jersey at Newark, Newark, New Jersey, US. ${ }^{2}$ Hospital Central Militar, Secretaría de la Defensa Nacional, México, Distrito Federal, México. ${ }^{3}$ Hospital Ángeles de León, León, Guanajuato, México. ${ }^{4}$ Residente de Cirugía General, Escuela Militar de Graduados de Sanidad, Universidad del Ejercito y Fuerza Aérea, México, Distrito Federal, México.

\section{RESUMEN.}

Objetivo. El análisis de la destrucción urbana masiva, como aquella ocurrida el 11 de septiembre de 2001 en Manhattan, Nueva York, Estados Unidos de América, nos permite conocer la naturaleza y comportamiento de estos hechos y organizar la distribución de los recursos humanos y materiales para planear la mejor solución al problema.

Fuentes. Búsqueda bibliográfica y a través de la Internet de información epidemiológica reciente relativa a la destrucción urbana masiva.

Resultados. De 5 a $26 \%$ por ciento de los pacientes evaluados en los Departamentos de Urgencias necesitan ingreso hospitalario. Las principales causas de ingreso son lesiones por inhalación y quemaduras; el trauma mayor multisistémico es raramente observado. De los pacientes admitidos, 11-13\% requieren tratamiento quirúrgico y 6-17\% requieren cuidados críticos, la mayoría (88\%) para ser sometidos a ventilación mecánica. Entre el 5 y $15 \%$ de los pacientes presenta un valor del índice de severidad (ISS) $>15 ;<2 \%$ presenta un ISS $>25$. La mortalidad global de los eventos de destrucción urbana masiva es de $16-25 \%$, con una tasa de mortalidad inmediata (en el lugar del desastre) de 90-99\%.

Conclusiones. A pesar del hecho de que estos eventos generan un número masivo de víctimas, sólo una pequeña proporción requiere tratamiento médico de alta especialidad. Es importante colaborar para mantener la integridad física de los rescatadores.

(Rev Biomed 2005; 16:159-167)

Palabras clave: destrucción urbana masiva, tratamiento intrahospitalario.

Solicitud de sobretiros: Mayor M.C. Luis M. García-Núñez. Circuito Naranjeros No. 22, Colonia Club de Golf México, Tlalpan, C.P. 14620, Distrito Federal, México. Teléfono 5573-0026, Fax 5573-0026.

E-mail: luismanuelgarcianunez@yahoo.com,lmgarcian@hotmail.com

Recibido el 2/Septiembre/2005. Aceptado para publicación el 7/Septiembre/2005.

Este artículo está disponible en http://www.uady.mx/sitios/biomedic/revbiomed/pdf/rb051632.pdf

Vol. 16/No. 3/Julio-Septiembre, 2005 
LM García-Núñez, R Cabello-Pasini, R Padilla-Solís, P Garduño-Manjarrez.

SUMMARY.

Anatomy of the World Trade Centre, New York, USA, disaster from an epidemiological perspective. What should we learn from massive urban destruction?

Objective. The analysis of massive urban destruction, as occurred on September 11, 2001, in Manhattan, New York, United States of America, allows to us to determine the nature and behavior of these facts and organize the distribution of the human and material resources for planning the best solution to the problem. Source of data. Bibliographic and "on-line" research of current information related to epidemiology of massive urban casualties.

Results. From 5 to $26 \%$ of patients evaluated in Emergency Departments require hospital admission. Major causes of hospital admission are inhalation injuries and burns; major multisystemic trauma is rarely seen. Among these admitted patients, $11-13 \%$ require surgical treatment and 6-17\% are treated in Critical Care Units, most of them (88\%) are undergoing mechanical ventilation. Between $5-15 \%$ of patients present injury severity scores (ISS) $>15 ;<2 \%$ of them show a score $>25$. Overall mortality among patients in case of massive urban destruction is $16-25 \%$; immediate mortality rate (on the disaster setting) is 90 $99 \%$.

Conclusions. Despite the fact these events generate a large number of casualties, only a small proportion of them require high-specialty medical treatment. It is of critical importance to collaborate in maintaining the physical integrity of the rescue workers.

(Rev Biomed 2005; 16:159-167)

Key words: Massive urban destruction, intra-hospital treatment.

\section{INTRODUCCIÓN.}

La clara y brillante mañana del 11 de septiembre de 2001 parecía transcurrir de manera cotidiana en el bajo Manhattan, Nueva York, E.U. Sin embargo, a las 8:46 horas, las calamidades comenzaron a ocurrir. El avión del vuelo 11 de American Airlines, un jet transcontinental Boeing 767, se impactó contra la torre norte de las Twin Towers (Torres Gemelas) del World Trade Center (WTC), y a las 9:03 horas, 17 minutos después, el correspondiente al vuelo 175 de la misma línea aérea comercial hizo impacto contra la torre sur $(1,2)$.

Las Torres Gemelas clásicamente se conocían como albergue para 50,000 empleados; cerca de 200,000 personas transitaban en sus pisos diariamente, más de la mitad eran turistas. Ocupaban un área de piso de 12 millones de pies cuadrados; la torre sur tenía una altura de $415 \mathrm{~m}$ y la norte de 417 $m$ (1). El peso combinado de ambas torres era estimado en poco más de 1.5 millones de toneladas. Había en ellas 43,600 ventanas, 16 millones de escalinatas y 20,000 puertas de elevador $(1,2)$.

Se cree que al momento del ataque había cerca de 17,000 personas en el complejo del WTC $(1,2)$. Cada Boeing 767 liberó una cantidad de casi 34,000 litros de combustible hacia los edificios, lo cual hizo alcanzar una temperatura dentro de ellos de $750^{\circ} \mathrm{C}$. La torre sur se vino abajo 57 minutos después del choque y la torre norte 29 minutos más tarde. Cada edificio se colapsó en cerca de 10 segundos, a una velocidad estimada de $80 \mathrm{~km} / \mathrm{h}$. Una vez derrumbadas, las torres quedaron reducidas a una masa de escombros de $50 \mathrm{~m}$ de altura.

La magnitud subjetiva del desastre es conocida por todos; sin embargo, los números son claros y escalofriantes: hubo 2,726 personas muertas, casi todas fallecidas en ese mismo día; sólo 13 de estas personas vivieron poco más de 24 horas. De las 1,450 personas que se encontraban en los pisos superiores al choque de los aeroplanos, sólo cuatro sobrevivieron $(1,2)$.

EL DESARROLLO DE LOS HECHOSY LA CONDUCTA DE UN CENTRO URBANO DE TRAUMA.

Existen cinco hospitales cercanos al área donde se encontraban las Torres Gemelas: NYWeill Cornell Medical Center, Downtown Hospital, Beth Israel Medical Center, Bellevue Hospital Center y St. Vincent's Hospital (1). Este último figuró como el receptor primario de los pacientes que provenían de la zona de desastre, ya que se encuentra a menos de 
una milla del sitio donde se dio el ataque. La experiencia en la atención intrahospitalaria proporcionada por este Hospital durante el desastre, reportada por Kirschenbaum y col. (2), representa un documento único para la comunidad médica interesada en la planeación y logística de la atención masiva de las víctimas de trauma y su análisis es, sin duda, un pilar de los fundamentos que para la institución de lineamientos de atención en pacientes traumatizados, en un ambiente de destrucción urbana masiva, pudieran formalizarse en el futuro (3).

Es importante conocer la capacidad del $S t$ Vincent's Hospital para entender la conducta que se observó durante los sucesos (2). Es un hospital de 550 camas, con un total de 48 camas de cuidados intensivos. Provee de un servicio de Cuidados Críticos, de forma permanente, por medio de cuatro médicos especialistas en el área. Su infraestructura da cabida a cinco médicos (Fellows) para efectuar la subespecialidad de Cuidados Intensivos.

A las 8:46 horas del día 11 de septiembre de 2001, en respuesta al "Sistema de Dirección para Incidentes", se activó hospitalariamente el "Código 3", lo cual alertó a la planta de médicos que el "Plan de Manejo de Emergencia Externa" había sido puesto en marcha. A los 15 minutos de la activación del código, todas las cirugías electivas, sin excepción, se habían cancelado, y se tuvieron a entera disposición 10 quirófanos y dos tomógrafos listos para la recepción de los pacientes. A las 9:05 horas (dos minutos después del accidente en la torre sur), había instalados en el Departamento de Urgencias (DU) 42 unidades de atención aguda, cada una con un médico y una enfermera, y una unidad de lavado ocular, la cual posteriormente tuvo que reubicarse debido a que el espacio en el DU fue insuficiente. Se habilitaron, asimismo, 73 unidades más en la sala de recuperación anestésica, cirugía ambulatoria, unidad de hemodiálisis, salón de endoscopia y laboratorio de cateterización cardiaca.

Mientras tanto, en el terreno, se instalaron múltiples áreas de "Triage" por la Agencia Federal de Manejo de Emergencia. Dichas áreas fueron cubiertas por médicos provenientes de cada uno de los Hospitales mencionados previamente (2, 3). A las 9:21 horas, todos los puentes y túneles de Nueva York habían sido cerrados en ambas direcciones. La zona al sur de la $14^{\text {th }}$ Street fue controlada enteramente por las autoridades gubernamentales y se denominó "zona congelada" ("frozen zone"). A esta zona, los hospitales aledaños enviaron los apoyos que fueron demandados por los médicos del terreno.

Adyacente a la entrada del DU, se instalaron tres equipos de apoyo médico consistentes cada uno en 300 médicos, 100 enfermeras y 500 elementos hospitalarios de profesión variada. Sin embargo, la localización de estos equipos tuvo que ser cambiada hacia una escuela cercana, debido a que estos sitios improvisados no dieron abasto a la cantidad de gente que demandaba atención. Es importante notar que con el colapso de la torre norte a las 10:28 horas, se suspendieron los servicios públicos del bajo Manhattan (2), lo cual afecto grandemente el desempeño de los Hospitales (3), pues algunos de ellos tenían servicios compartidos con el área de desastre.

$\mathrm{Al}$ inicio del plan de atención, la utilización de las camas fue puesta bajo el control de Director Médico del Hospital, la Dirección de Operaciones y el Director de la Oficina de Estadística. En conjunto, evaluaron la necesidad y disponibilidad de camas de Cuidados Críticos, así como la disponibilidad del staff para atender estas unidades. De esta manera, se facilitó la conversión de unidades de atención rutinaria a unidades provisionales de cuidados críticos, por medio de la provisión de medios humanos y materiales necesarios. Esa misma mañana, se dieron de alta 50 pacientes de salas generales para tener más camas a disposición. Los médicos especialistas en Cuidados Críticos se asignaron para cubrir cada una de las cuatro unidades de Terapia Intensiva con las que cuenta el Hospital, y se les pidió que seleccionaran 15 pacientes para ser manejados en las salas de cuidado general médico-quirúrgico.

El Director del Departamento de Urgencias y el Jefe del Servicio de Trauma fueron los encargados iniciales del "Triage" (2). Los equipos de trauma estaban listos para esperar y recibir a los pacientes en 


\section{LM García-Núñez, R Cabello-Pasini, R Padilla-Solís, P Garduño-Manjarrez.}

la ambulancia y caminar directamente al DU. Cada equipo de trauma estaba dirigido por un cirujano general del staff.

El arribo de las personas lesionadas comenzó varios minutos después del ataque (el primer paciente proveniente del sitio de desastre ("campo cero" o "Ground Zero") arribo al St. Vincent's Hospital a las 10:30 horas) y alcanzó el pico dos a tres horas después $(2,3)$. Se seleccionó a los pacientes con las lesiones más graves para dirigirlos directamente al DU: 1) pacientes con mecanismo de lesión por caída mayor de ocho pies o rodamiento por escaleras, 2) trauma contuso o penetrante, 3) lesiones por quemadura o por explosión, 4) dificultad respiratoria, 5) dolor torácico, 6) alteraciones en el estado de conciencia y 7) fracturas centrales. Una vez estabilizados en el DU, los pacientes fueron trasladados al quirófano o a la Unidad de Cuidados Intensivos, donde el equipo de Cuidados Críticos se encontraba esperándolos. Los pacientes con otro tipo de lesiones y los heridos que podían caminar, fueron enviados a centros de valoración satélites, ubicados, como se mencionó, inicialmente en las afueras del DU y posteriormente en una escuela.

De los 1,688 pacientes valorados en el servicio de Urgencias, 1,103 fueron supervivientes tratados por afecciones o lesiones relacionadas con el ataque $(2,3)$. El promedio de la edad de los pacientes fue de 39 años, y $66 \%$ fueron del sexo masculino. Veintiséis por ciento de los pacientes llegaron al DU transportados por vehículos de Emergencia Médica y $29 \%$ por vehículos de rescate improvisados. Setenta $\mathrm{y}$ tres por ciento de los pacientes fueron tratados $\mathrm{y}$ egresados del DU. Entre los supervivientes con lesiones, $49 \%$ tuvieron lesión por inhalación y $29 \%$ trauma ocular. Dieciséis por ciento de los supervivientes requirieron hospitalización; más de un tercio de los pacientes (37\%) padecieron lesiones por inhalación, $19 \%$ presentaron quemaduras de distinta gravedad y $0.4 \%$ murieron durante su atención de urgencias.

Cincuenta por ciento de los pacientes que fueron valorados y admitidos a través del "Triage" se presentaron en las primeras cuatro horas posteriores al accidente. El Hospital admitió 78 pacientes el primer día, dos tercios de los mismos en las primeras dos horas posteriores al desastre $(2,3)$. En total, se admitieron 112 víctimas del ataque, $24 \%$ de todas las víctimas admitidas en los Centros Hospitalarios que participaron en la atención de estos pacientes. Las principales causas de admisión fueron: lesión por inhalación (37\%), quemaduras (19\% pacientes), trauma múltiple/lesión grave por aplastamiento (13\%), desórdenes psiquiátricos $(9 \%)$, lesiones musculoesqueléticas de distinta magnitud $(8 \%$ pacientes) y enfermedades cardiovasculares de reciente presentación o crónicas agudizadas (8\%). Veinticuatro por ciento de los pacientes admitidos tenían condiciones no traumáticas relacionadas con el ataque (agudización de enfermedades cardiovasculares o psiquiátricas). Ocho por ciento de los pacientes fueron admitidos a Unidades de Cuidados Críticos, de los cuales $88 \%$ requirieron ventilación mecánica. Uno de estos pacientes murió por traumatismo craneoencefálico severo (3).

En las primeras 48 horas post-desastre, se realizaron 13 procedimientos operatorios a las víctimas de trauma, los cuales consistieron principalmente en laparotomía exploradora. Los debridamientos y las reducciones cerradas se programaron en pequeños espacios eventuales para mantener la disponibilidad del salón de operaciones.

El St. Vincent's Hospital (2) reportó una onda inicial grande de supervivientes, la cual alcanzó un pico 3 a 4 horas después de que el primer paciente llegó al Hospital. El DU evaluó aproximadamente 300 pacientes en las primeras dos horas, pero esto disminuyó aproximadamente a 20 pacientes después de la cuarta hora (3). En estos momentos, se presentaron los primeros casos de solicitud de atención médica por parte del personal de los rescatadores.

A las 4 de la tarde del día 11 de septiembre, las áreas satélite de cuidados críticos ya estaban despejadas, y no se encontraban recibiendo pacientes. El DU registro 426 pacientes el primer día, y más de 240 adicionales vistos en el servicio de lavado ocular. Se estima que cerca de 800 víctimas del ataque fueron evaluadas ese día en el hospital. En el periodo inicial,

\section{Revista Biomédica}


la mayoría de los pacientes presentaron lesiones por inhalación y oculares. Las quemaduras corneales químicas relacionadas con los componentes del concreto fueron muy comunes. Cinco por ciento de los pacientes que se presentaron al DU tuvieron un Indice de Severidad de la Lesión (Injury Severity Score o ISS) $>15$. Menos de $2 \%$ de los pacientes tuvieron un $I S S>25$. Estos pacientes sufrieron principalmente lesiones por aplastamiento, fracturas múltiples y trauma contuso mayor. En este grupo (ISS $>25$ ) ocurrieron sólo tres muertes tempranas, ninguna de las cuales se esperaba sobreviviera. Una de estas fatalidades fue debida a lesión por inhalación grave, y dos por trauma contuso masivo. Quince pacientes tuvieron quemaduras de $2^{\circ} \mathrm{o} 3 \mathrm{er}$. grado. Cuatro de estos pacientes presentaron afectación de más de $85 \%$ del área de superficie corporal total y requirió manejo por una unidad especializada de quemados en Nueva York. Por supuesto, también se dieron víctimas del trauma emocional: una persona presentó dolor torácico de inicio súbito tras haber observado el colapso de las torres. Fue trasladada al St. Vincent's Hospital y tuvo que ser intervenida de urgencia por disección aguda de un aneurisma de la aorta torácica.

En las siguientes $48 \mathrm{~h}$, sólo unos pocos pacientes requirieron admisión al St. Vincent's Hospital por lesiones relacionadas con el ataque $(2,3)$. Estas lesiones ocurrieron principalmente en rescatadores que continuaban laborando en el "Campo Cero". Las afecciones que más comúnmente presentaron estos pacientes fueron dificultad respiratoria, lesiones ortopédicas menores, y síndrome de estrés postraumático. Hubo algunos casos de quemaduras en las extremidades inferiores por falta de protección adecuada, ya que entre los escombros se mantenía una temperatura extremadamente alta.

La recolección de datos demográficos (2) y clínicos (3) de las víctimas atendidos en los hospitales previamente mencionados comenzó seis horas después del ataque, e incluyó todos aquellos casos desde las 8 de la mañana del 11 de septiembre a las 8 de la mañana del 13 de septiembre.

Varios problemas logísticos impactaron el desempeño del trabajo hospitalario $(2,3)$. La electricidad y el agua corriente del bajo Manhattan, así como las comunicaciones por telefonía celular y computación, se vieron suspendidas. La comunicación tuvo que ser mantenida por medio de radios portátiles. El cierre de las vías de acceso a Manhattan también impidió inicialmente el aporte de suministros y el traslado del personal. Sin embargo, posteriormente, las autoridades se mostraron flexibles para minimizar esta situación.

\section{DISCUSIÓN.}

Los desastres en masa pueden provenir de varios entornos. Uno de ellos es la destrucción urbana masiva (DUM), situación que puede deberse a fenómenos naturales (terremotos, maremotos), confrontaciones bélicas o terrorismo (2). En la Ciudad de México, la experiencia más próxima que ejemplifica este escenario, aunque no debida a un ataque terrorista, es el terremoto del 19 de septiembre de 1985.

El Sistema de Salud Pública juega un papel fundamental en la atención de las víctimas de DUM. Son los hospitales públicos los sitios en los cuales se concentraran los medios humanos y materiales para encarar la situación (3). A pesar de que en un inicio la magnitud del desastre es desconocida y parece lógico emplear todos los recursos con los cuales se cuenta, el aporte desmedido de ellos puede acarrear desgracias tardías por dispendio y gasto inadecuado de los mismos, dando lugar a una carencia posterior.

A lo largo de la historia, se ha visto que la DUM, independientemente de la causa, observa algunos puntos de consistencia y que son comunes a una gran variedad de escenarios. Por ejemplo, en 1983, durante el ataque a las Fuerzas Armadas de los Estados Unidos en Beirut, Líbano (4), 70\% de los elementos en el sitio del accidente fueron lesionados. Noventa y siete por ciento de los fallecimientos fueron de manera inmediata; de los supervivientes, sólo $17 \%$ presentaron lesiones críticas. En la explosión en el Murrah Federal Building en Oklahoma en 1995 (5,6), 88\% de las personas presentes en el lugar fueron lesionadas. Cuarenta y cinco por ciento de los pacientes lesionados murieron. Noventa y ocho por ciento de 


\section{LM García-Núñez, R Cabello-Pasini, R Padilla-Solís, P Garduño-Manjarrez.}

las muertes fueron inmediatas y en el lugar del suceso; sólo $11 \%$ de los pacientes que sobrevivieron presentaron lesiones críticas y fueron admitidos al Hospital. Thompson y col. (7) reportaron que durante el ataque terrorista en las Torres de Khobar, Arabia Saudita, 73\% de las personas presentes en el lugar resultaron lesionadas, $16 \%$ de los pacientes atendidos en el Departamento de Urgencias fueron hospitalizados y $6 \%$ de los mismos requirieron cuidados críticos; la tasa de mortalidad fue de $25 \%$. Arnold y col. (8), en una extensa revisión de la literatura, reportaron 8,364 victimas en 29 ataques terroristas de DUM por instrumento explosivo, con una tasa de mortalidad de $8 \%$, dividida en tres fases. Más del 90\% de las muertes ocurrieron inmediatamente, en el lugar del accidente. La tasa de supervivencia inmediata tras el suceso fue de $89 \%$. Veintiséis por ciento de los pacientes lesionados requirieron atención en Departamentos Hospitalarios de Urgencias, de los cuales se hospitalizó el $66 \%$.

Severance (9) señala que la gran mayoría (8590\%) de las víctimas que sufren lesiones en casos de DUM, presentan lesiones que no ponen en peligro la vida y que incluso son capaces de desplazarse por sí mismas. Esto significa al mismo tiempo, que 5-15\% de las víctimas necesitarán atención médica de urgencia, o incluso, cuidados críticos.

Aunque el número absoluto de muertes fue mayor en los ataques al WTC que en los reportes previos de DUM en la literatura médica, las proporciones mencionadas con anterioridad se mantienen relativamente conservadas. Hubo 2726 muertes debidas al atentado en las Torre Gemelas, dando sobre el estimado de cerca de 17,000 personas en el complejo al momento del ataque, una tasa de mortalidad de $16 \%$. De estos fallecimientos, $99.5 \%$ se dieron en el lugar del accidente o en las primeras 24 horas, $0.5 \%$ de los fallecimientos ocurrieron después del primer día. En el St. Vincent's Hospital (2), se evaluaron 1688 casos ese día, 1103 por afecciones directamente relacionadas con el ataque. Cerca de 800 pacientes (51\%) fueron vistos en las primeras 24 horas. De éstos, $27 \%$ fueron vistos en las primeras 2 horas (3).
La mayoría de los pacientes fueron hombres jóvenes, con un promedio de edad de 39 años y laboralmente activos $(2,3)$. Entre los supervivientes del ataque que llegaron al Hospital, la mayoría presentó lesión por inhalación (49\%) y trauma ocular de distinta magnitud (29\%). Setenta y tres por ciento de estos pacientes fueron evaluados y egresados; $16 \%$ fueron hospitalizados por presentar principalmente lesión por inhalación grave $(37 \%)$, quemaduras de distinta gravedad (19\%), y trauma múltiple combinado con lesiones por aplastamiento (13\%). Es importante notar que sólo $5 \%$ de los pacientes admitidos tenía un ISS $>15$, y menos del $2 \%$ de los pacientes mostraron un ISS $>25$.

Ocho por ciento de los pacientes admitidos requirió manejo en las Unidades de Cuidados Intensivos (2,3). Ochenta y ocho por ciento de estos pacientes requirió manejo con ventilación mecánica. Se requirió cirugía de Urgencia en $11 \%$ de los pacientes admitidos. Hubo una tasa de mortalidad de $3 \%$ entre los pacientes hospitalizados (4 de 112).

En un reporte similar proveniente de la experiencia de dos hospitales comunitarios de Manhattan, durante ese mismo ataque terrorista del 11 de septiembre de 2001, Cushman y col. (3) reportaron una incidencia que $85 \%$ de los pacientes atendidos en el Departamento de Urgencias fueron víctimas de daños menores (inhalación de sustancias e irritación ocular). La tasa de admisión hospitalaria fue de $15 \%$ y $13 \%$ de los pacientes admitidos requirió cirugía. El ISS medio para los pacientes atendidos en este escenario fue de 12. La tasa de mortalidad hospitalaria fue de $0.4 \%$.

En casos de DUM, la coordinación entre las autoridades es de suma importancia. El "Sistema de Dirección para Incidentes" es una herramienta administrativa muy útil en caso de contingencias médicas (1-3), la cual fue desarrollada en California, E.U., en 1970, y que tiene por objeto establecer un contacto inicial y formal entre la autoridad que se encuentra manejando la contingencia en la zona de desastre y aquella que se encargará de subsanar algunas necesidades. Durante los eventos del 11 de septiembre de 2001, este sistema mostró su utilidad

\section{Revista Biomédica}


al permitir una óptima colaboración entre la Policía de Nueva York y los hospitales de la zona. El sistema consiste en dar aviso a una autoridad superior (por ejemplo, el presidente del Hospital), el cual deberá entablar y mantener contacto permanente, así como garantizar el funcionamiento constante y girar instrucciones de empleo a los siguientes elementos: 1) Oficina de Apoyo Legal, 2) Oficina de Información Pública, 3) Oficina de Control de Personal, 4) Oficina de Abastecimientos y Logística, 5) Oficina de Estadística, 6) Oficina de Dirección de Operaciones, 7) Director Médico, y 8) Oficina de Información al paciente y la familia.

El registro estadístico es extremadamente importante para sustentar con bases firmes la disposición de los medios.

\section{CONCLUSIONES.}

La DUM es un evento que pone a prueba los Sistemas de Salud Pública (2,3). La disposición de los recursos humanos y materiales juega un papel clave en la atención a las víctimas del suceso, pues existe el riesgo de que los medios disponibles se agoten o hagan falta en los momentos más críticos, lo cual podría empeorar la ya caótica situación.

Para disponer de los recursos, es importante conocer la naturaleza de los eventos de DUM. Característicamente, éstos ocasionan un gran número de víctimas (2), los cuales pueden alcanzar centenas, o aun millares $(3,10)$. Sin embargo, sólo una pequeña proporción de los mismos requerirán atención médica de alta especialidad y demandarán medios avanzados de tratamiento (11).

De la experiencia proveniente de reportes previos de eventos de DUM, y de la atención hospitalaria provista por los Centros de Trauma de Manhattan durante los hechos acaecidos el 11 de septiembre de 2001, se nos permite concluir algunos puntos que pueden servir como una orientación muy general de las características que observan estos eventos. Del 70 al $85 \%$ de las personas presentes en el lugar del desastre, sufrirán algún grado de lesión (2, $3,9,11) ; 85 \%$ a $95 \%$ de estas víctimas mostrarán lesiones que no ponen en peligro la vida. La mortalidad global de los eventos de DUM es del 16 al 25\% (10, 11); $90 \%$ a $99 \%$ de los casos de mortalidad ocurren en el lugar del accidente; la tasa de muertes tardías (entre el día 1 al 14 post-desastre) oscila entre 0.5$3 \%$; la mortalidad de los pacientes lesionados durante su atención en el DU es cercana al $0.4 \%$. La mortalidad entre los pacientes que requieren cuidados críticos es de 17 a $25 \%$ (9-11).

Del 5-26\% de los pacientes lesionados requieren atención hospitalaria. La mayoría de los pacientes son hombres jóvenes, sin patología crónica agregada. Durante la DUM, las causas más comunes de demanda de atención hospitalaria de urgencia son la irritación ocular y las lesiones por inhalación. A manera de guía, cerca del $50 \%$ del total de pacientes que el Hospital recibirá durante el curso del evento se verán en el primer día (2); de éstos, $50 \%$ serán valorados en las primeras 2 horas (3). Setenta a setenta y cinco por ciento de los pacientes pueden ser egresados del servicio, con instrucciones domiciliarias de tratamiento. Cinco a $20 \%$ de las víctimas de DUM presentan un $I S S>15$, y cerca del $2 \%$ un $I S S>25$ (12); la mayoría de estos pacientes presentan lesiones por aplastamiento, fracturas múltiples y trauma mayor contuso.

Dieciséis a veinticinco por ciento de los pacientes valorados en el DU requerirán admisión hospitalaria, aunque existen reportes aislados de tasas de admisión tan altas como de $66 \%(2,3)$. Las causas más comunes de ingreso hospitalario en casos de DUM son: lesiones por inhalación, quemaduras y trauma múltiple/lesiones por aplastamiento. El trauma sistémico mayor es infrecuentemente visto en los pacientes provenientes de escenarios de DUM, pues casi todos ellos sucumben en el desastre (12).

De los pacientes que ingresan, 11-13\% requerirán tratamiento quirúrgico, casi siempre de urgencia. Del 6 al 17\% de los pacientes admitidos ameritan tratamiento en Unidades de Cuidados Críticos, siendo la indicación más frecuente la necesidad de ventilación mecánica ( $90 \%$ de los casos) (2).

Anexo al fenómeno generado dentro del hospital, es importante no hacer a un lado algunos otros rubros, 


\section{LM García-Núñez, R Cabello-Pasini, R Padilla-Solís, P Garduño-Manjarrez.}

también notables y que acompañan invariablemente a la atención de las víctimas de $\operatorname{DUM}(2,3,9,11)$. Habitualmente, los servicios de Urgencias son abarrotados y el espacio físico se ve sobrepasado por la afluencia de gente, pacientes y familiares, que acuden a solventar sus demandas. La política de atención médica de urgencia del St. Vincent's Hospital recomienda fuertemente que el "Triage" debe ser dirigido por un médico con amplia experiencia en la atención del trauma, y no debe ser practicado en el interior del DU, sino en las afueras del mismo, para evitar saturar de personal el área. Es importante evitar el sobre-Triage, ya que se ha visto que la calidad de atención médica hospitalaria de los pacientes víctimas de trauma, disminuye notablemente, con un impacto sensible en el pronóstico, cuando la frecuencia de flujo para intervención primaria mayor excede los 4-5 casos por hora. La colocación de puestos provisionales de atención médica temporal en las afueras del DU del St. Vincent's Hospital no demostró ser una buena idea, debido a que posteriormente se tuvo que buscar una construcción en forma para organizar los consultorios médicos. Probablemente sea conveniente instituir de forma primaria, un centro de atención médica temporal en una construcción formal.

Dado que la experiencia dicta que la gran mayoría de los pacientes durante la DUM solicita asistencia por presentar daño debido a inhalación de sustancias nocivas e irritación ocular, debe destinarse un amplio espacio específico para proporcionar terapia respiratoria u oxigenoterapia, así como para practicar lavado ocular. Esto redundará en facilitar el egreso de estos pacientes y despejar tan pronto como sea posible la zona de atención médica.

Después de un tiempo (habitualmente 4 a 6 horas, según lo sucedido en Manhattan), los rescatadores comenzaran a demandar tratamiento médico por lesiones ocurridas durante las actividades de socorro $(2,3)$. Estas lesiones habitualmente son menores (lesión por inhalación, irritación ocular, quemaduras y trauma ortopédico menor) y requieren una intervención mínima a moderada. Es importante recomendar a los rescatadores lesionados suspender sus labores para no entorpecer las maniobras y evitar progresión del daño, y reforzar específicamente las medidas de seguridad y protección en el área de socorro. Asimismo, el hospital debe estar preparado para un cataclismo secundario en la zona de desastre.

La agudización de problemas cardiovasculares (hipertensión arterial y coronariopatías) y psiquiátricos, es la regla más que la excepción en casos de DUM (2). Deben adoptarse las medidas necesarias para que estos pacientes sean tratados como víctimas no traumáticas de la DUM.

El hospital debe tomar en cuenta que los servicios públicos (agua, electricidad, gas, teléfono convencional y celular) se ven afectados ostensiblemente durante los desastres urbanos (2), y es indispensable coordinar una efectiva provisión de los mismos en caso de que no estén disponibles de manera normal.

Por último, nunca estará de más mencionar la enorme importancia que la labor estadística reviste en este tipo de eventos $(2,3)$. La recolección de los datos deberá iniciarse en cuanto las condiciones lo permitan, idealmente, unas pocas horas después del suceso. Una información precisa permitirá dirigir los esfuerzos y los recursos hacia las áreas que se perciban como críticas, y de esta forma, maximizar el aprovechamiento y mejorar el pronóstico de las víctimas de tan lamentables desgracias.

\section{REFERENCIAS.}

1.- Wang D, Sava J, Sample G, Jordan M. The Pentagon and 9/11. Crit Care Med 2005; 33 S:S42-S7.

2.- Kirschenbaum L, Keene A, O’Neill P, Westfal R, Astiz ME. The experience at St. Vincent's Hospital, Manhattan, on September 11, 2001: Preparedness, response, and lessons learned. Crit Care Med 2005; 33 S: S48-S52.

3.- Cushman J, Pachter HL, Beaton HL. Two New York City hospitals' surgical response to the September 11, 2001, terrorist attack in New York City. J Trauma 2003; 54:147-55.

4.- Frykberg ER, Tepas J, Alexander R. The 1983 Beirut Airport terrorist bombing: injury patterns and implications for disaster management. Am Surg 1989; 55:134-41.

5.- Mallonee S, Shariat S, Stennies G. Physical injuries and fatalities resulting from the Oklahoma City bombing. JAMA 
1996;276:382-7.

6.- Maningas PA, Robison M, Mallonee S. The EMS response to the Oklahoma City bombing. Prehosp Disaster Med 1997; 12:80-5.

7.- Thompson D, Brown S, Mallonee S, Sunshine D. Fatal and non-fatal injuries among U.S. Air Force personnel resulting from the terrorist bombing of the Khobar Towers. J Trauma 2004; 57:208-15.

8.- Arnold JL, Halpern P, Tsai MC, Smithline H. Mass casualty terrorist bombings: a comparison of outcomes by bombing type. Ann Emerg Med 2004; 43:263-73.

9.- Severance HW. Mass-casualty victim "surge" management. Preparing for bombings and blast-related injuries with possibility of hazardous materials exposure. NCJM 2002; 63:242-6.

10.- Frykberg ER. Disaster and mass casualty management: a comment on the ACS position statement. Bull Am Coll Surg 2003; 88:12-3.

11.- Peral-Gutiérrez de Cevallos J, Turégano-Fuentes F, PérezDíaz D, Sanz-Sánchez M, Martín-Llorente C, Guerrero-Sanz JE. 11 March 2004: the terrorist bomb explosions in Madrid, Spain - an analysis of the logistics, injuries sustained and clinical management of casualties treated at the closest hospital. Crit Care 2004; 8:in press.

12.- Hirshberg A. Managing mass casualties: a no-nosense approach. Guiding problems. Proceedings of the Trauma Surgery and Surgical Critical Care Meeting, Las Vegas, Nevada, March 2005; 1:25-33. 